\title{
Learning Analytics in Small-Scale Teacher-Led Innovations: Ethical and Data Privacy Issues
}

\author{
María Jesús Rodríguez-Triana \\ REACT \\ École Polytechnique Fédérale de Lausanne (EPFL) \\ Lausanne, Switzerland \\ maria.rodrigueztriana@epfl.ch \\ Alejandra Martínez-Monés \\ GSIC-EMIC \\ Dpto. Informática, Universidad de Valladolid \\ Valladolid, Spain \\ Sara Villagrá-Sobrino \\ GSIC-EMIC \\ Dpto. Pedagogía, Universidad de Valladolid \\ Valladolid, Spain
}

\begin{abstract}
As a further step towards maturity, the field of learning analytics (LA) is working on the definition of frameworks that structure the legal and ethical issues that scholars and practitioners must take into account when planning and applying LA solutions to their learning contexts. However, current efforts in this direction tend to be focused on institutional higher education approaches. This paper reflects on the need to extend these ethical frameworks to cover other approaches to LA; more concretely, small-scale classroomoriented approaches that aim to support teachers in their practice. This reflection is based on three studies where we applied our teacher-led learning analytics approach in higher education and primary school contexts. We describe the ethical issues that emerged in these learning scenarios, and discuss them according to three dimensions: the overall learning analytics approach, the particular solution to learning analytics adopted, and the educational contexts where the analytics are applied. We see this effort as a first step towards the wider objective of providing a more comprehensive and adapted ethical framework to learning analytics that is able to address the needs of different learning analytics approaches and educational contexts.
\end{abstract}

Keywords: Learning analytics, ethics, privacy, higher education, primary education

\section{INTRODUCTION}

The increasing trend towards massive data collection in educational settings has raised new ethical concerns in the learning analytics (LA) research community. On the one hand, there is a need to identify students across platforms and retrieve as much data as possible in order to obtain informed analysis about the learning processes. On the other hand, other aspects influence the adoption and acceptability of learning analytics approaches, such as data ownership and openness, potential abuse and the need for new key competences to interpret and act on learning analytics results 
(2016). Learning analytics in small-scale teacher-led innovations: Ethical and data privacy issues. Journal of Learning Analytics, 3(1), 43-65. http://dx.doi.org/10.18608/jla.2016.31.4

(Greller \& Drachsler, 2012). These and other aspects are part of the ethical concerns that the field of learning analytics must face in order to grow as a mature discipline.

Several authors have reflected on the ethical issues that affect the field (Slade \& Prinsloo, 2013; Sclater, 2014) and have made proposals to deal with them, like the set of design guidelines proposed by Pardo and Siemens (2014). However, most of these analyses and proposals apply to higher education institutional contexts. Little reflection has been done on the implications of using learning analytics in smaller-scale contexts where teachers use the data to manage their university or schoollevel classrooms, especially on the latter. However, as pointed out by Griffiths (2012), the kind of ethical considerations that must be taken into account are different depending on the approach taken to learning analytics. Therefore, there is a need to reflect on what ethical aspects are relevant in the applications of learning analytics to small-scale teaching practices, and especially in school contexts.

During recent years, we have worked on the support of teachers' orchestration (Dillenbourg, 2013) of CSCL activities in blended learning scenarios. With the aim of providing teachers with meaningful and easy-to-appropriate data, we have proposed both scripting and monitoring processes, each aware of the other, so that the analysis and results provided by the learning analytics system are influenced by the information provided (by the teacher) at design time (Rodríguez-Triana, MartínezMonés, Asensio-Pérez, \& Dimitriadis, 2012; Rodríguez-Triana, Martínez-Monés, Asensio-Pérez, \& Dimitriadis, 2013). Both processes are supported by a technological infrastructure - a data integration architecture - able to integrate different kinds of data sources, including LMS, tools, and user-generated data (Rodríguez-Triana, Martínez-Monés, \& Asensio-Pérez, 2011). This integration aims to apply learning analytics to the existing technological ecosystems with which the users (teachers and students) are familiar.

These proposals were designed and validated following a design-based research (DBR) process applied to seven cases in higher education (Rodríguez-Triana, Martínez-Monés, Asensio-Pérez, \& Dimitriadis, 2015). To test the capability of our solutions to adapt to different educational levels, we applied them to a primary school case. We found that while we could easily manage the technical aspects of data retrieval, analysis, and visualization, emergent issues related to data ownership and control, student identity, and other legal and ethical concerns were more difficult to handle.

We present, in this paper, a reflection on how the ethical frameworks proposed in LA literature apply to our teacher-centred classroom-based approach and to the two aforementioned educational contexts. The two studies presented in the paper illustrate with concrete examples which ethical issues are relevant in learning analytics when applied to small-scale teacher-led innovation in higher education and at school levels. The findings of the studies provide initial evidence of the need to adapt existing ethical frameworks to the different approaches to learning analytics and to the educational contexts to which they may be applied. 
(2016). Learning analytics in small-scale teacher-led innovations: Ethical and data privacy issues. Journal of Learning Analytics, 3(1), 43-65. http://dx.doi.org/10.18608/jla.2016.31.4

The rest of the paper is structured as follows: Section 2 provides an overview of the ethical issues identified in the learning analytics literature; Section 3 describes the main aspects of our approach to LA; Sections 4 and 5 explain how we applied our learning analytics approach in university and primary school contexts as well as the main ethical issues that we encountered; Section 6 discusses and compares the findings obtained from the different contexts; and finally, Section 7 provides the main conclusions drawn from this work.

\section{ETHICAL ISSUES IN LEARNING ANALYTICS: A FRAMEWORK OF REFERENCE}

As the field of learning analytics emerges from its infancy, ethical issues related to its application to real practice are receiving more attention. In parallel with the increasing impact of the proposals coming from the field, there is a need to reflect on the consequences that these proposals have on the persons involved (Slade \& Prinsloo, 2013), and on how learning analytics must be shaped to respond to these demands (Pardo \& Siemens, 2014).

We aim to provide new insights on how ethical issues depend on the approach to LA, and on the particular contexts to which these approaches are applied. In order to structure the analysis, there is a need to organize the issues and establish a common framework that enables discussion about them (Ferguson, 2012). However, to the best of our knowledge, few frameworks yet address the ethical issues related to the application of small-scale teacher-led learning analytics in schools. There exist studies that draw attention to the potential benefits of learning analytics for primary schools (see e.g., Ebner \& Schön, 2013), or deal with how to support LA-enabled teacher interventions (Wise, 2014), but they do not usually address the ethical or privacy concerns that may affect them.

The existing frameworks take an institutional approach. Kay, Korn, and Oppenheimer (2012) identify a set of legal and ethical issues, with a focus on higher education. Sclater (2014) provides a comprehensive list of concepts and issues related to ethics, accompanied by an extensive literature review. Based on this review, and after a process of structured discussion and validation involving experts and stakeholders (Sclater, 2015), a Code of Practice for learning analytics was released to support educational institutions in the effective use of learning analytics (Sclater \& Bailey, 2015). In the rest of this section, we describe the categories defined in the Code of Practice, pointing out how the issues covered apply to the teacher-led small-scale learning analytics approach addressed in this paper.

Responsibility: There is a need to define who is responsible for the legal, ethical, and effective use of LA. Concretely, specific responsibility should be allocated for data collection, anonymization, analysis, retention, and stewardship, as well as intervention. At the classroom level, teachers will be, in many cases, the ones responsible for these aspects. One question derived from this is whether the teachers involved have the capacity if provided with the tools needed to face this responsibility. 
(2016). Learning analytics in small-scale teacher-led innovations: Ethical and data privacy issues. Journal of Learning Analytics, 3(1), 43-65. http://dx.doi.org/10.18608/jla.2016.31.4

Transparency: This aspect refers to how and whether students should be informed about data collection, results, and the methods of analysis. Any potential adverse consequences of withdrawal from a learning analytics process should also be explained to them. At the institutional level, it makes sense to ask whether teachers should be aware of this information, while in small-scale approaches, this question does not normally apply, as the teachers will be the ones to control the analysis. Therefore, the main concern in this case is whether and how the envisioned data collection and analysis are to be presented to the students.

Consent: This topic refers to how and in which circumstances students (parents) should be asked for consent to collect and use their data, e.g., aspects related to informed consent, whether students hold the right to opt out, and what the consequences of doing this will be for the individual and for the group. Consent can be formulated to enable students to decide on the data being collected about them, or to preserve their anonymity. This topic also includes issues related to the informed nature of the consent, asking whether consent can be considered as informed when the participants do not understand the analytics, or when these analytics may evolve in the future in unknown ways. The need to describe the analysis so that the participants understand it may be very relevant at the school level, where the participants (teachers, children, and their parents) may find it difficult to understand what is planned. At the classroom level, opting out may not be possible if the analysis forms part of the learning activities. In cases where it is possible, one issue to address is how to provide teachers with logistical support to exclude these learners from the analysis.

Privacy: Access to student data and analytics must be restricted to those with a legitimate need to view them. There are important issues related to the capacity of learning analytics algorithms to reidentify individuals based on the aggregation of these data sources. If there is a need to use "sensitive data" (e.g., ethnicity, religion, sexual preference) additional safeguards and possibly additional consent should be collected before granting any request from an external body to share data. Privacy may be less important at the classroom level, when data is only used and shared within the class. However, it is necessary to ensure that privacy is not being violated, especially when working with minors, where this is a very sensitive issue.

Validity: This concept refers to the extent to which the analysis can be trusted. Relevant questions relate to which data sources are necessary to ensure accuracy, how to verify the algorithms used to draw conclusions, and how to avoid drawing conclusions from spurious correlations. In contexts where learning analytics is applied to historical data, it is also necessary to consider that students develop, learn, and grow, and the conclusions based on that data must consider that development. Finally, this aspect refers to a more technical issue related to the authentication of data sources coming from public sites or, in general, from third parties, i.e., how to ensure that students are correctly identified when using data coming from these external data sources. In classroom-based experiences, where blended learning is common, there are many threats to validity, as participants may interact in many forms, including face to face outside the classroom. 
(2016). Learning analytics in small-scale teacher-led innovations: Ethical and data privacy issues. Journal of Learning Analytics, 3(1), 43-65. http://dx.doi.org/10.18608/jla.2016.31.4

Access: This topic relates to whether and in which ways students can access the analytics that make use of their data. This aspect considers the need to allow students to correct inaccurate data about themselves. At the small scale, teachers can ask the learners or consider extra data sources in order to make corrections.

Enabling positive intervention: This topic relates to what should be done by the institution with the information obtained, and what the consequences of not doing anything are, e.g., not informing a student that $s /$ he risks failing a subject. This aspect also includes issues related to pedagogical interventions, such as who should be responsible for presenting the results and how, the possible danger of favouring one group over another, how to adapt the intervention to the different needs of the students, et cetera. The approach discussed in this paper assumes that learning analytics is applied to support teacher interventions in the class. As with any other pedagogical intervention, it is necessary to reflect on the impact that these interventions (or lack thereof) have on the learners.

Minimizing impact: This topic covers some of the main adverse impacts already identified in the application of learning analytics and how to deal with them. We should be aware that an analysis can never give a complete picture of an individual's learning and may sometimes ignore personal circumstances. Thus, we must ensure that trends, norms, categorization, or any labelling of students does not create bias, reinforce discriminatory attitudes, or increase social exclusion. At the small scale, this aspect is closely related to the effect that the intervention will have on the students.

Stewardship of data: This aspect is related to the administration of data by the institution, including compliance with the legal requirements applicable in each case. Data for learning analytics must comply with existing institutional data policies, and these policies must ensure that the rights of all the involved persons are maintained. Additionally, only the minimum data required to deliver the purposes of the analytics should be collected and they should only be retained for appropriate and clearly defined periods. Teachers require help in accomplishing these tasks.

This classification, proposed by the Code of Practice, was useful to structure the discussion about ethical concerns found in the cases described in Sections 4 and 5. Moreover, this discussion helped us identify aspects where the framework should be adapted to the approach to learning analytics addressed in this paper. The next section outlines the main characteristics of this approach.

\section{OUR APPROACH TO LEARNING ANALYTICS}

Griffiths (2012) identifies two types of analytic interventions with potential impact on teaching practices: 1) methods oriented to achieve enhanced regulation of the teaching and learning environment; and 2) methods and tools intended to help lecturers carry out their tasks more effectively. Our approach to learning analytics is aligned with the second approach. It is devoted to supporting teacher reflection, as opposed to prediction, to use the terms of Greller \& Drachsler (2012). This section outlines the main components of our approach, highlighting those aspects of the 
(2016). Learning analytics in small-scale teacher-led innovations: Ethical and data privacy issues. Journal of Learning Analytics, 3(1), 43-65. http://dx.doi.org/10.18608/jla.2016.31.4

proposed solutions with an impact on the ethical issues discussed in this paper.

Our learning analytics approach is designed to provide monitoring information to be used for regulation, formative assessment, or self-reflection about the learning design and the learning process. We aim to provide teachers with feedback about the accomplishment of pedagogical decisions made at design time. The educational settings where the approach can be applied cover face-to-face and distance activities carried out at different social levels (individual, group, and whole class). In other words, blended Computer-Supported Collaborative Learning (CSCL). In addition, in terms of technological support, the proposal is devoted to distributed learning environments (DLEs) made up of an institutional virtual learning environment (VLE), Web 2.0 tools, and GLUE! - an architecture used to integrate third-party tools into VLEs. As described in the rest of this section, our work was designed to address specific challenges met in these contexts that also have ethical implications. These challenges included the need to support teachers in taking responsibility for data analysis and interpretation; how to help them with stewardship of data in these complex technological systems; as well as how to enhance data validity in blended learning supported by DLEs.

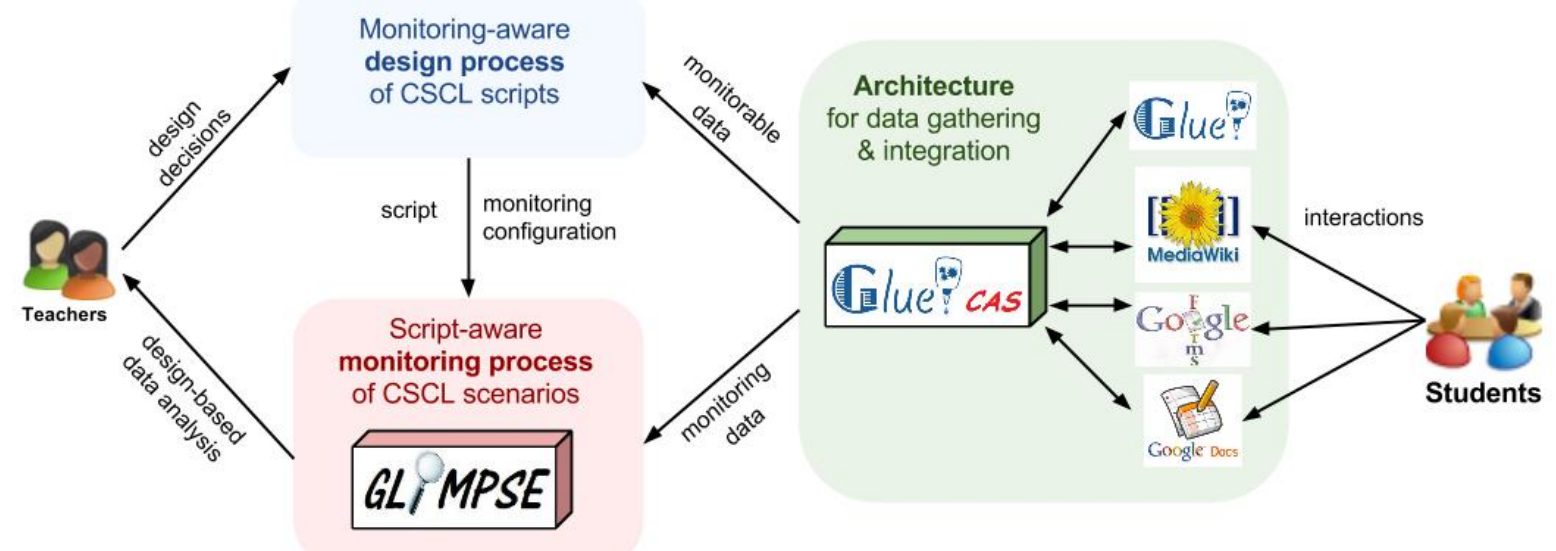

Figure 1: Overview of the main components involved in our learning analytics approach.

The solution consists of three components (See Figure 1): two processes and an architecture for data gathering and integration in DLEs to help teachers integrate learning analytics within their practice. The first component is a monitoring-aware design process of the learning scenario that takes into account the teacher's information needs (Rodríguez-Triana et al., 2013). This enriched design process helps teachers identify and make explicit which moments or aspects of their envisioned learning activities should be monitored. These aspects are based on the identification of a set of constraints: special conditions that must be met to comply with the pedagogical intentions expressed in the design, e.g., whether participation of all the students in a group is required in a certain phase of the activity, or whether a particular product must be delivered at a specific deadline, et cetera. The process entails a second phase, during which teachers are prompted to 
(2016). Learning analytics in small-scale teacher-led innovations: Ethical and data privacy issues. Journal of Learning Analytics, 3(1), 43-65. http://dx.doi.org/10.18608/jla.2016.31.4

enrich the design to satisfy these monitoring needs (e.g., choosing the most appropriate tools for both pedagogical and monitoring concerns, or identifying complementary data sources). The second component of the solution is a monitoring process guided by the decisions made at design time (Rodríguez-Triana et al., 2012). In this monitoring process, the data gathering is focused on those sources chosen by the teacher and the data analysis is designed to verify whether the current state (the gathered evidence) matches the desired state (the learning design). These two processes (design and monitoring) are two sides of an overall approach that aims to help teachers take control of the data analysis and interpretation. By means of the design process, they become active participants in the definition of the analysis. The contextualized visualization of the results enabled by the script-aware monitoring process is designed to support teachers in their interpretation of the analyses.

The third component of our proposal is GLUE!-CAS, an architecture that addresses the need of data gathering and integration in DLEs made up of VLEs (typically Moodle or Mediawiki) and Web 2.0 tools (e.g., Google applications) (Rodríguez-Triana et al., 2011). GLUE!-CAS defines how to collect and integrate data coming from these data sources, overcoming the problems of gathering data from external tools when using a VLE. However, in blended scenarios, these automatic data sources are not enough to get a full picture of the interactions taking place. Frequently, part of the learning process occurs outside the technological context. Besides, the information and communication technologies (ICTs) register a limited set of evidence, usually based on user interactions with the platforms, and sometimes it is not possible to authenticate the student identity properly (Slade \& Prinsloo, 2013). Since restricting the analysis to the data registered by the ICT tools may offer only a partial view of the user activity (Avouris, Fiotakis, Kahrimanis, Margaritis, \& Komis, 2007), GLUE!-CAS enables the integration of ad-hoc information provided by the participants in the learning context. This practice enriches the evidence gathered and allows teachers to triangulate the data coming from the different data sources. From an ethical point of view, this approach enables teachers and students to correct the data automatically collected from the ICT tools (Sclater, 2014) and to better understand the reasons behind the results obtained (Greller \& Drachsler, 2012). Overall, this integration of heterogeneous data sources is designed to increase the validity of the analysis. In terms of privacy, this involvement of the stakeholders also has benefits, letting them decide what information they want to share (Slade \& Prinsloo, 2013). Finally, the technical solution helps teachers to face the difficulties associated with data stewardship in DLEs.

To support teachers in their analysis, we implemented GLIMPSE (Rodríguez-Triana et al., 2013), a tool that, interacting with GLUE!-CAS, automates data gathering, integration, and analysis, offering the teacher a comparison between the current and the desired state of the learning scenario. The product of this tool is a monitoring report where the information is visualized by means of tables that connect the participants, the data sources, the indicators, and the warnings that emerged from the analysis (see Figure 2 below for an example). 
(2016). Learning analytics in small-scale teacher-led innovations: Ethical and data privacy issues. Journal of Learning Analytics, 3(1), 43-65. http://dx.doi.org/10.18608/jla.2016.31.4

\section{FIRST SCENARIO: HIGHER EDUCATION}

The proposal presented in the previous section was iteratively applied to seven learning scenarios in higher education (Rodríguez-Triana et al., 2015). In this paper, we focus on the last two of these scenarios, which were designed to evaluate the proposal in its final version. This section offers an overview of the learning contexts as well as how the proposal was implemented throughout the learning scenario lifecycle. We will not describe the results related to the application of the learning analytics approach as such, which have been reported elsewhere (see Rodríguez-Triana et al., 2015). We will focus on illustrating those aspects of the cases that had an influence on the ethical issues described in Section 2.

\subsection{Learning Context}

The approach described in Section 3 was applied to two learning scenarios in higher education with a common profile: 3-4 weeks, implementing learning designs inspired by CSCL principles and supported by DLEs, interweaving face-to-face and distance activities as well as blended interactions among students. The studies involved two teachers from different backgrounds, with different levels of expertise in CSCL scenarios, and different knowledge about the proposal. To reference these studies, we will use HE1 and HE2 as labels for the first and second scenarios in higher education.

The first study (HE1) was carried out in an Early Childhood Education course, involving a teacher who was not expert in CSCL scenarios and who had less than 6 years of teaching experience. Of the 165 students enrolled in the course, 150 participated. The learning scenario lasted 4 weeks and consisted of various distance and face-to-face activities combining individual and collaborative work. The whole learning design was supported by Moodle and Google applications, and involved a total of 316 resources. The main challenge of this scenario was to cope with the high number of students and resources.

The second study (HE2) took place in an educational research course that formed part of a Master's degree for pre-service secondary education teachers. The teacher in charge of this course was an expert in CSCL and had previous knowledge of the approach. Over 3 weeks, 15 students were involved in this study. They worked on defining a proposal for an educational research project, combining individual, group, and class activities, as well as face-to-face and distance learning. The whole learning process was technologically supported by means of MediaWiki and Google applications, requiring 77 files in total. The main challenge of this scenario was the complexity of the design: many interrelated activities occurring in a short period of time that demanded much attention from the teacher to avoid problems that could jeopardize the scenario.

\subsection{Application of the Proposal}

The application of the proposal started with the design of the learning scenarios, following the 
(2016). Learning analytics in small-scale teacher-led innovations: Ethical and data privacy issues. Journal of Learning Analytics, 3(1), 43-65. http://dx.doi.org/10.18608/jla.2016.31.4

monitoring-aware design process (see Section 3). This process is designed to give responsibility of the learning analytics process to the teacher and provides a means of addressing the validity of the analysis. Besides paying attention to the pedagogical aspects of the learning activities, the design process supported the teachers' articulation of their monitoring concerns. Based on the information provided in the design, and following the proposed design process, the teachers were informed about the data available to verify the constraints of the envisioned learning scenario, whether there was enough evidence to evaluate them, and which complementary data sources could be used to improve the analysis. This information gave the teachers the opportunity to reflect on the monitoring process and enhance it. In both scenarios, the teachers decided to triangulate the data coming from the ICT tools with data provided ad-hoc by the students and the teachers themselves. These aspects are illustrated in the reflections made by both teachers when they were interviewed after the design phase:

It has helped me to first reflect about which were the most conflictive points of the design. Once they were identified, and knowing which technological tools I was going to use in each phase, I could know both what kind of information they would provide and what the best moment to get that information would be. [...] In the original design, I did not foresee controlling classroom attendance in a systematic manner, nor administering a questionnaire to find out the collaboration, task distribution, and workload aspects for each of the phases. [...] The design process made me appreciate the importance of including new information sources to enable gathering additional evidence. [HE1, Teacher interview after the design phase]

In case the tools that I had in mind did not provide monitoring information, I would have substituted them with other tools (provided they have similar functionality to support the students' work, of course). [HE2, Teacher interview after the design phase]

Thus, throughout the monitoring-aware design process, the teachers were responsible for the data gathering and analysis, defining which constraints needed to be evaluated during the enactment, selecting the data sources, the information to be retrieved, and when it should be collected.

A second ethical aspect addressed in the proposal regards transparency and consent. Once the teachers defined the analysis to be carried out, the students were informed about the data to be gathered, the metrics, the purpose of the analysis, and its potential impact. We then asked them to give their permission for data collection to take place: almost all the students participating in the learning scenarios agreed to this (150 out of 165 in HE1 and all 15 in HE2). The tools supporting the design process allowed us to specify which students were taken into consideration, focusing the analysis on those students who had given their consent. Therefore, our proposal was able to address, at least from a technical point of view, the problems associated with letting learners opt out from the analysis.

A third issue relates to the stewardship of data. During the scenarios, the students' data regarding the actions registered on the different platforms (e.g., accesses, editions, uploads, et cetera) was 
(2016). Learning analytics in small-scale teacher-led innovations: Ethical and data privacy issues. Journal of Learning Analytics, 3(1), 43-65. http://dx.doi.org/10.18608/jla.2016.31.4

gathered from the technological environment. The data came from the VLEs (Moodle and MediaWiki), the Web 2.0 tools (e.g., Google Apps), and the architecture that supported the integration of the tools in the VLEs (GLUE!). On the one hand, both the VLEs and GLUE! were hosted on our own servers. Thus, we did not have to deal with permission issues in order to connect the data gathering tool (GLUE!-CAS) with these platforms, which normally constitutes an obstacle when working with institutional or third-party platforms. On the other hand, GLUE!-CAS allowed us to collect the data from the third-party Web 2.0 tools integrated in the DLE. Thus, even if the information was initially controlled by the owners of these tools, we made it available for the teachers to use for their analyses. The integration of these data by GLUE!-CAS enabled teachers to control these data, and use it even when it was removed or no longer available in the original tools.

The integration of data in GLUE!-CAS is also important to increase the validity of the analysis. As mentioned above, this focus on validity is addressed by the design process, which promotes teacher reflection on the accuracy of the data and enables them to include new data sources to enhance this validity. Following these design decisions, teachers and students contributed actively by providing their own data. The teachers provided information about student attendance at the face-to-face sessions together with a few notes, and the students, by means of questionnaires, described how they had participated in the learning activities (how they had interacted, which tools were used, how much time they had devoted to the tasks, et cetera).

Via GLUE!-CAS and GLIMPSE, data generated by the multiple data sources were collected, integrated, and analyzed according to the teacher's decisions. Based on the data gathered, the teachers received the corresponding monitoring reports taking into account the relevant data sources, the actions to be logged, the timeframes, and the indicators to be verified. Error! Reference source not found. shows one of these monitoring reports sent to the teacher (only three groups appear due to constraints of space). The columns show, from left to right: the groups and their members, the data sources employed in this case, and the warnings issued by GLIMPSE when a condition specified at design time is not met. The cells coloured in green refer to students or activities that have complied with the specified constraints, while the red cells highlight the points where there is no evidence supporting the teacher's expectations about the students' involvement or the use of resources. For example, Figure 2 shows that no access by StudentName 6 to the Final research proposal had been registered. The teacher had specified at design time that this resource had to be used at least once by each group member, as the lack of access could imply a lack of participation of that member in the authorship of the report. This problem was visualized in the report by means of the red cell corresponding to StudentName6, and the warning that appears on the right-hand side of the table. As can be observed, the reports summarized the evidence collected, highlighting potential problems that needed to be checked. 
(2016). Learning analytics in small-scale teacher-led innovations: Ethical and data privacy issues. Journal of Learning Analytics, 3(1), 43-65.

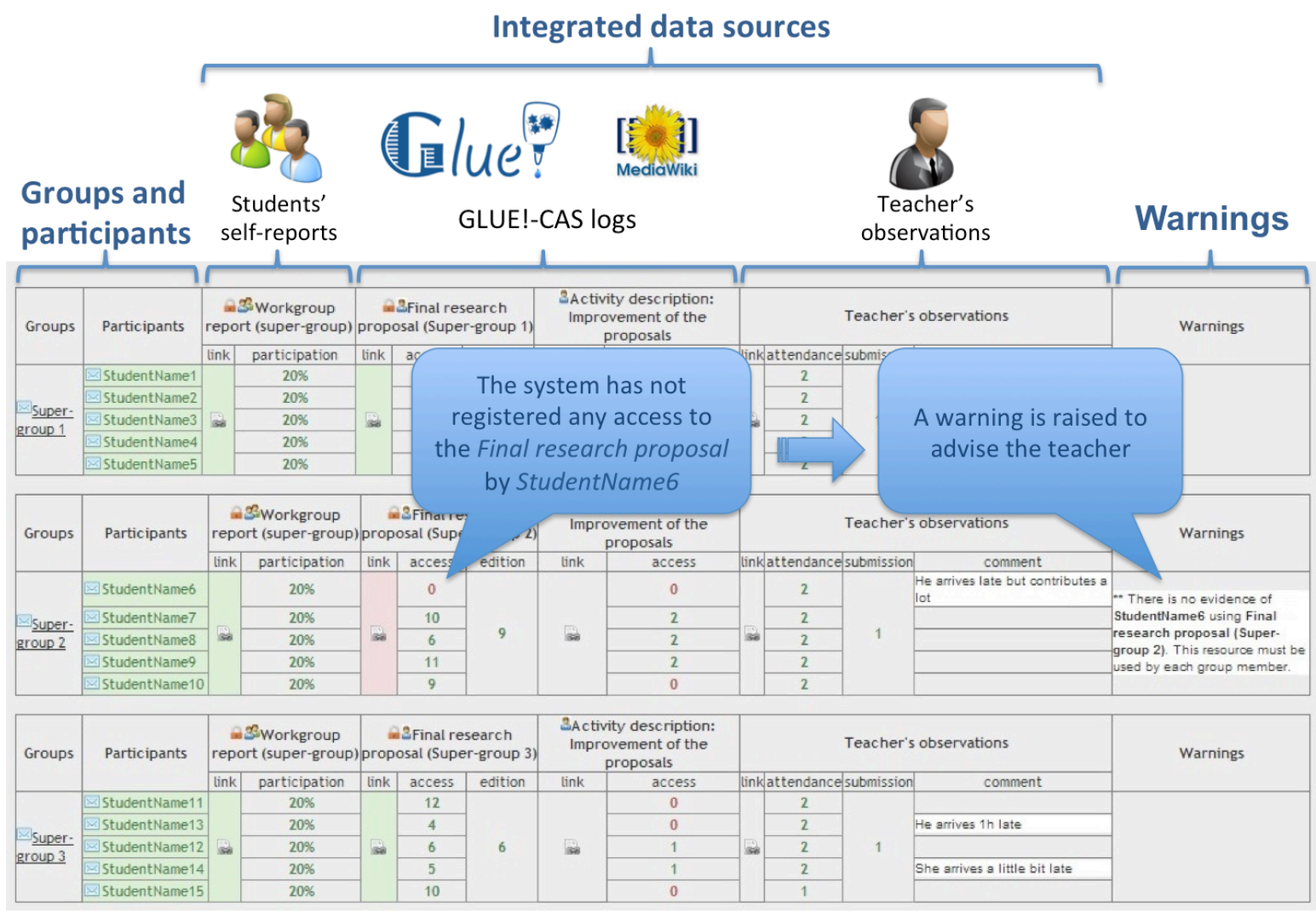

Figure 2: Excerpt from the monitoring report sent to the teacher the HE2 study (anonymized version).

The integration of multiple data sources in the analysis played a crucial role in the success of the proposal. First, it significantly increased the accuracy of the results. Complementing the evidence from the ICT tools with the teacher's observations and the students' feedback allowed us to take into account not only the computer-mediated interactions but also the face-to-face ones.

The students' report has been very useful, in order to take into account their perspective. [...] Being able to incorporate my notes with the comments received from students has simplified my work a great deal. [...] Having all the data gathered in one place simplifies the monitoring. Having the information centralized helps avoid misunderstandings and keeps the situation under control. [HE1, Teacher interview after the enactment]

The integration is not only useful, it is necessary. It gives you a complementary view of an activity that happens in different settings/moments. It uses blended sources to inform blended activities. [HE2, Teacher interview after the enactment]

We evaluated the validity of the monitoring reports by comparing the results obtained with the complementary teacher observations, the additional student comments, the researcher observations, and the learning outcomes in the tools. Despite the existence of errors, the monitoring 
(2016). Learning analytics in small-scale teacher-led innovations: Ethical and data privacy issues. Journal of Learning Analytics, 3(1), 43-65. http://dx.doi.org/10.18608/jla.2016.31.4

reports provided a perspective of the learning process that was close to the real facts (in $99.67 \%$ and $97.81 \%$ of the evaluated conditions).

As previously discussed, our approach hands responsibility for the analysis (both the design and the interpretation) to teachers. One aspect that must be addressed regarding responsibility is whether teachers are able to appropriate the analyses and act upon them. As can be seen in Figure 2, the information provided to teachers was direct and contextualized in their designs. Both teachers agreed that interpreting the reports could be done quickly and did not present any problems.

Interpreting the monitoring reports was very easy. With one look, I knew whether there had been any problems. [...] I dedicated 10 minutes at most: 5 minutes to read everything, plus another 5 minutes to take the corresponding measures. [HE1, Teacher interview after the enactment]

Interpreting the reports was simple and immediate. The information provided is clear and does not lead to misinterpretations. [HE2, Teacher interview after the enactment]

During the learning scenario, the teachers used the information to identify potential problems as well as to intervene and regulate the scenario when appropriate. Afterwards, once the activities ended, the teachers employed the monitoring reports to support reflection on the learning process.

When I detected a problem, I contacted the student. In fact, I have sent quite a few emails with wake up calls, reminders, and asking what happened. This is something we normally do not do because we do not have means to follow the students' work closely. [HE1, Teacher interview after the enactment]

The monitoring reports required regulatory tasks only in two cases. In general, all students/groups followed the plan, except for the development of the report in small groups (which I commented on verbally with them in the classroom) and in the last activity, in which three students forgot to send the peer-assessment report, and thus I had to send a reminder to them via email. It was critical because neither they nor I would have remembered until the final assessment, which coincided with the end of the course. The rest served to check that everything was going well. [HE2, Teacher interview after the enactment]

Regarding student access to the data analysis, we should remember that our approach is devoted to supporting teachers. Thus, during the scenarios students did not automatically have access to the monitoring reports. However, the messages sent to the teacher in the reports made it clear that the results had to be checked. Therefore, any non-expected result should have been discussed with the student, who could clarify the reasons behind it. The teachers' comments during the interviews presented earlier illustrate how they proceeded in such cases.

In summary, the two university cases illustrate how our classroom-based learning analytics approach 
provides teachers with tools to become responsible for data analysis; how data stewardship was solved by the use of GLUE!-CAS (and the fact that we could use our own servers at the university); how we dealt with the need to provide transparent information to the participants; and how the technical solution enabled teachers to exclude from the analysis those who did not sign the consent form. We have also shown our emphasis on the validity of the analysis by means of the integration of several data sources, planned at design time and addressed technically by GLUE!-CAS; and how the results were shown by GLIMPSE in a contextualized manner that teachers could appropriate to intervene in the learning situations when needed. The students did not receive the data directly, but teachers were prompted to compare results with other available information before acting, as the whole proposal makes teachers aware of the weaknesses associated with data analysis in blended scenarios.

\section{SECOND SCENARIO: PRIMARY SCHOOL}

As we described in Section 3, our proposal was designed to help teachers in blended CSCL scenarios supported by DLEs. Although all our previous studies had been carried out in higher education, we realized that at other educational levels, teachers faced similar problems. Thus, we set out to apply our proposal in a primary education setting with the aim of checking its adaptability to this new context, and to unveil the potential limitations and constraints imposed by such a learning context. This section provides a brief description of the learning context including an analysis of the different ethical issues we faced during the study.

\subsection{Learning Context}

The case study was run in a first grade class (6-7 years old) with 24 students at a rural school in Valladolid, Spain. This school has 300 students and 29 teachers. The teacher involved in this study used Blogger in his classes in combination with external Web 2.0 tools (such as YouTube videos or Educaplay games). In spite of a general positive experience with this approach, one of his main concerns was that these blogs did not tell him who had read or accessed the pages. This precluded him from knowing whether the students were using the learning materials offered in the blog.

In order to address this problem, we used GLUE! to build the DLE. Blogger was treated as the VLE where the third-party tools (e.g., the videos and games) were embedded. With this technological setting, it was possible to trace student activity during the learning process and then inform the teacher about their use of the tool.

The intervention ran between 14 May and 2 June 2014. The teacher proposed two lesson plans involving a blog and several external resources, in which the teacher asked the students to carry out some activities at home such as watching a video, reading an online text, or playing educational games. 
(2016). Learning analytics in small-scale teacher-led innovations: Ethical and data privacy issues. Journal of Learning Analytics, 3(1), 43-65. http://dx.doi.org/10.18608/jla.2016.31.4

\subsection{Application of the Proposal}

As with the previous study, during this design process, the teacher described the learning activities, chose the resources to be used, and configured the ways in which the learning scenario would be monitored: he decided which constraints should be checked, specified student actions to be monitored, as well as the timeframes and delivery dates of the monitoring reports.

Once the teacher configured the analyses, we informed the different participants, namely the school principal and the parents/legal guardians of the students, since they were not adults. A detailed explanation of the data, metrics, purpose, and potential impact of the analyses was given to them. Both the school principal and the families supported this kind of innovative practice and gave their consent for the data collection.

Regarding the collection of identified data, we encountered an unexpected legal obstacle. Blogger can be configured so that only registered users access the blogs, but this requires a Gmail account. However, 6-7 year old children are not legally allowed to own an email account on that server. In order to overcome this obstacle, we had to look for a workaround, asking the families to open an email account on behalf of their children, and then access the site using that account. Even so, we did not find it difficult to obtain consent and family support to create and use the dummy e-mail accounts. However, we must note that obtaining consent when working with minors is a very delicate issue and must be carefully addressed.

Another issue we encountered related to the kind of data that could be collected and analyzed. In upper educational levels, the input and interactions with technologies may be very rich. However, at the age of 6 to 7, students frequently do not have the skills to carry out very complex tasks (e.g., the students wrote little before the end of the course). Thus, the kind of data available is likely to be limited, and many online educational tools do not offer any data about the learner's work. In fact, the data gathered from the technological environment mainly consisted of the actions logged by GLUE!, specifically student accesses to the Web 2.0 tools. This information was easily accessible because GLUE! was hosted on our servers and we could access the systems without additional bureaucracy.

While the learning scenario was taking place, the teacher received monitoring reports according to the plan drawn up at design time. Figure 3 shows a piece of information from those monitoring reports. These reports simply presented the evidence collected (accesses to the tools) highlighting potential problems that needed to be checked, e.g., students who had not accessed the tools, and therefore, could not have used the resources. The monitoring report presented in the figure refers to the number of times the students accessed the activities in one of the monitored lesson plans. It can be seen that only three students of 24 completed all the activities, while nine out of 24 did not access any. The same kind of visualization was shown to the teacher for the second lesson plan. The results relating to the participation of the students were similar. Only five students out of 24 ISSN 1929-7750 (online). The Journal of Learning Analytics works under a Creative Commons License, Attribution - NonCommercial-NoDerivs 3.0 Unported (CC BY-NC-ND 3.0) 
(2016). Learning analytics in small-scale teacher-led innovations: Ethical and data privacy issues. Journal of Learning Analytics, 3(1), 43-65. http://dx.doi.org/10.18608/jla.2016.31.4

completed all the activities, while 11 out of 24 did not access any. When the researchers shared these results with the teacher, he felt confused, as we can see in the following extract:

Honestly, I did not think this could happen. I mean, I thought that the majority of my students could complete the activities. I think it is important to communicate these results to the families. [PE, Teacher interview after the enactment]

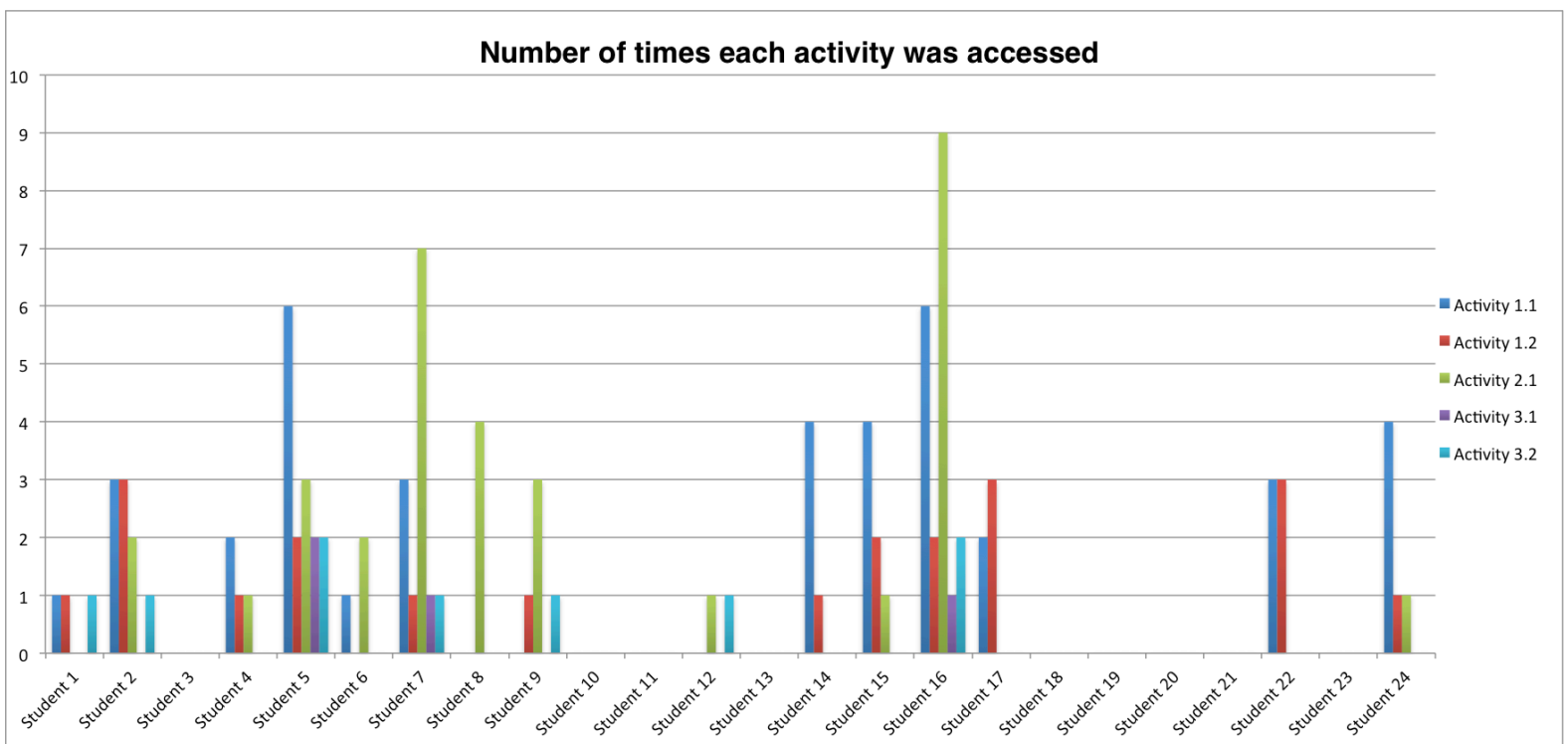

Figure 3: Output shown to the teacher about the number of students who accessed the activities in Lesson Plan 1.

During the face-to-face sessions, the teacher asked the students whether they had done their homework in order to compare their answers with the monitoring reports. The information was generally accurate and helped the teacher to intervene appropriately. However, one of the issues that we faced related to the students' virtual identities. As noted by the teacher in the second interview, not all families knew how to solve some of the technical difficulties, and some families accessed the site using the parents' own accounts.

When I met with the families, many of them told me that they had had many problems accessing the activities embedded in the blog. They said that they had read and followed all the instructions I gave, but still found it impossible. Moreover, I noticed when I reviewed the monitoring report, that there were families that had accessed the activities with a different e-mail account. [PE, Teacher interview after the enactment]

Obviously, the technical difficulties encountered by the families, together with the workaround we had to use to be able to identify the children using surrogate accounts, resulted in a case of "enmeshed identities" (Greller \& Drachsler, 2012). Aware of this problem, in the final interview, the teacher said that if he were to use a similar activity in future, he would devote more time to training the families to access the on-line activities with the e-mail login, as well as supervising their usage. 
(2016). Learning analytics in small-scale teacher-led innovations: Ethical and data privacy issues. Journal of Learning Analytics, 3(1), 43-65. http://dx.doi.org/10.18608/jla.2016.31.4

In spite of the simplicity of the project and all the restrictions related to the quality of the data, the teacher was very positive about the feedback provided:

It is important to provide students with other ways of learning, as well as to assess learning. I can say that these teaching and learning practices are innovative for students and we have the opportunity to motivate them and engage the families in the educational process. [PE, Teacher's reflective diary]

In addition, the teacher identified several potential uses of the analysis. Among other uses, he highlighted knowing in advance whether the students had done their homework, being able to send reminders, identifying more/less attractive resources, detecting which days of the week the students were more active, analyze the students' development in relation to their homework, et cetera.

Regarding student access and privacy, we should recall that the teacher is the target user of the approach presented in this paper. Nevertheless, the results, especially in the case of unexpected behaviours, should be checked with students/parents who can clarify what happened. Especially in this case, the meaning of the reports might not be easily understandable by children due to their age. But the teacher could share it with the parents or with the institution itself as "evidence" of the learning process to be discussed. Therefore, teachers are in charge of sharing the data analyses with those who legitimately need to view them, and this sharing must take into account the privacy and legal issues applicable in each case.

\section{DISCUSSION}

The studies presented in Sections 4 and 5 illuminate our discussion about how the current frameworks related to ethical issues in learning analytics apply to the analytical interventions we envision, as well as how they depend on the educational contexts where we applied them. We identify three levels of discussion: 1) implications related to the learning analytics approach to which this work belongs, i.e., small-scale analytics to help teachers manage their classes; 2 ) implications derived from the actual solutions we propose to support this learning analytics approach, and 3) issues related to the educational contexts where the solutions are applied. After the discussion at these three levels, we drafted a set of guidelines that could be taken into account by teachers aiming to conduct learning analytics scenarios of the kind discussed in this paper.

\subsection{Implications Related to the Teacher-Centred Classroom-Based Approach to LA}

As mentioned above, our approach to learning analytics is oriented to supporting teacher reflection in order to help them with the management of their classrooms. This is one of the possible approaches to learning analytics identified by Griffiths (2012). The other two approaches are 1) institutional approaches that seek to increase the efficiency of the institution; and 2) approaches associated with the enhanced regulation of the teaching and learning environment. 
(2016). Learning analytics in small-scale teacher-led innovations: Ethical and data privacy issues. Journal of Learning Analytics, 3(1), 43-65. http://dx.doi.org/10.18608/jla.2016.31.4

As mentioned in Section 2, the framework used to structure our discussion responds to an institution-led approach. This focus can be observed in the kind of questions posed to analyze the concepts, and in the responsible stakeholders identified. The questions included in the framework focus on what the role of the institution and/or the students in the overall process of learning analytics is. Teachers appear only as secondary actors or - marginally - as potential providers of data. In contrast, in our approach the teacher plays the role of the institution - in control of the data and the analysis - and of the receiver of the information - role-played by the students in the reference framework.

Due to these differences, we had to adapt the questions posed in the framework to better fit our approach. This translation was straightforward. However, the need for adaptation could be an obstacle for a practitioner who wanted to employ the framework to analyze a learning analytics intervention of the kind discussed in this paper. This justifies the need to refine the ethical framework to adapt it to the characteristics of the learning analytics approach.

A first element of refinement would be the inclusion of teachers as responsible stakeholders, and of questions related to their role as data providers and receivers. A second element regards the emphasis given to different aspects of learning analytics. For example, while privacy issues are paramount in an institutional approach, they have different implications in a closed system, where the teacher uses already available data and shares it with the students. In fact, in these cases, new ethical issues may arise, more related to classroom orchestration, which should also be identified and included in the revised framework.

\subsection{Implications of the Design-Aware Learning Analytics Approach}

Our approach to learning analytics is strongly based on the participation of the teacher from the beginning of the lifecycle of the learning activity. It takes into account the need to integrate data from different sources - including data provided by teachers and students - that offer a complementary view to the evidence recorded by the technological infrastructure. This designaware learning analytics approach helps to address a number of ethical issues common in these contexts.

First, the approach has a strong impact on the validity of the data in these blended learning scenarios based on the use of DLEs. The integration of different kinds of data by means of the GLUE!CAS architecture, enriched the analysis. The fact that the design process helps teachers reflect on the missing data and enables them to introduce data provided by the participants also increased the validity. The results of the two cases show that the output given to the teachers, based on this combination of data, was accurate in most cases.

The second issue addressed by our proposal refers to responsibility for the analysis. The approach 
(2016). Learning analytics in small-scale teacher-led innovations: Ethical and data privacy issues. Journal of Learning Analytics, 3(1), 43-65. http://dx.doi.org/10.18608/jla.2016.31.4

helps teachers take decisions about the data and the analytics that will be applied to the setting. This schema makes teachers fully aware of the purpose of the analysis, and avoids possible negative effects of systems that demand new competencies of teachers in order to interpret the results.

The possibility of adverse impact is minimized because, in our case, the analysis does not entail any negative effect on the students. Indeed, the way the approach is presented to teachers makes them realize that the information available is not complete, and they are responsible for asking or looking for other data sources to complement it. Thus, no decision is made automatically based on the results. The analysis only aims to detect potential problems in order to address them as soon as possible. To be more precise, it is the teacher who, aware of the information, should verify the situation and intervene if necessary.

\subsection{Issues that Depend on the Educational Context}

Our experience in applying learning analytics to two different educational levels (see Sections 4 and 5 ) showed us that ethical issues also depend on the kind of learning analytics processes that can be expected in these contexts. As highlighted by the cases discussed in this work, we found new legal and ethical concerns in the primary school context that we had not met in higher education scenarios.

A first issue affected by this change of context refers to the stewardship of data. In higher education settings, it is usual the institution that owns the servers where the learning environments reside and the activities take place. Teachers can access these platforms, and count on technical staff to configure the analysis. On the contrary, schools often do not have easy access to this kind of learning platform, or, if they do, they do not have access to technical staff who can support the analysis (Blackwell, Lauricella, Wartella, Robb, \& Schomburg, 2013; Lim, Zhao, Tondeur, Chai, \& Tsai, 2013). Due to these problems, teachers and schools are increasingly relying on cloud and Web 2.0 tools. The dependency on these external Web 2.0 tools poses new legal problems and ethical issues, as was illustrated in our school case. Students (minors) cannot legally have their own accounts, leading to a conflict with the need for learning analytics processes to identify users. One solution, as we described, is to rely on the families of the children to obtain a surrogate identity, but this adds more complexity to the analysis and new threats to data validity. In the case discussed in this paper, some families were not able to help their children to perform the tasks, while others used the parents' identities to access activities.

In both cases, this had an effect on the validity of the data collected and displayed to the teacher. As discussed in the previous section, the teacher knew in advance the limitations of the implemented approach, and did not take any direct action based exclusively on the monitoring reports. Nevertheless, he found the information provided useful, as it gave him a general overview about how the students worked at home with the virtual environment that he had set up. 
(2016). Learning analytics in small-scale teacher-led innovations: Ethical and data privacy issues. Journal of Learning Analytics, 3(1), 43-65. http://dx.doi.org/10.18608/jla.2016.31.4

When working with lower level courses (like the first graders studied in this paper), children are not autonomous in their use of computers, and families must be considered actors to take into account at many levels (permission, training, collection of data, et cetera). The inclusion of families requires a reflection on how this change affects the other ethical issues discussed in the field. For example, the option of showing the results to the students - and therefore to their families - must be balanced against issues such as privacy, transparency, and action, as these are bound to be an issue when the families are aware that the tasks carried out at home are being analyzed and used by the teachers.

A final issue relevant for the application of learning analytics in school contexts is the simplicity of the interactions between the children - especially first graders - and the system. This poses some questions about the kind of inferences that can be drawn from this very basic information. In principle, considering the limitations imposed by the data available, a simple learning analytics approach showing basic data to the teacher seems more coherent than sophisticated analyses. However, future developments in the field could challenge this view.

Overall, our analysis suggests that the work being done by the learning analytics community to define a general framework or code of practice for learning analytics must take into account the approach and the educational contexts where learning analytics is applied. Further work on analyzing the particular characteristics common to the different approaches and educational contexts would help to identify these new aspects and to refine the proposed frameworks.

\subsection{Proposals for an Expanded Ethical Framework for Learning Analytics}

As a conclusion of our reflection, we propose a set of recommendations to be taken into account to extend or complement the existing frameworks of ethical issues in learning analytics. As discussed above, the existing frameworks are oriented to institutions and therefore apply to large and medium-scale institution-led learning analytic approaches. If we consider that learning analytics can be applied in small-scale scenarios, there is a need to adapt the existing frameworks to these approaches currently coexisting in the learning analytics field. A possible way to address this goal is to define different itineraries depending on the approach to learning analytics, so that practitioners can focus their view on the questions most important to their approach. A similar approach is taken in Kay et al., (2012), defining the different legal and ethical priorities in six use cases related to learning analytics in higher education.

One of these itineraries could be defined for the learning analytics approach discussed in this paper, i.e., classroom-based learning analytics oriented to supporting teachers' regulation of their classes. In order to provide an initial proposal, we have adapted the questions posed by Sclater's (2015) framework to produce a set of guidelines that could be taken into account by teachers willing to apply learning analytics from this perspective. These guidelines are presented in Table 1, ordered using the categories employed in the current Code of Practice (Sclater \& Bailey, 2015). 
(2016). Learning analytics in small-scale teacher-led innovations: Ethical and data privacy issues. Journal of Learning Analytics, 3(1), 43-65. http://dx.doi.org/10.18608/jla.2016.31.4

Contrary to the framework proposed by Sclater (2015), these recommendations assume that, in this context, teachers will take responsibility for most of the aspects mentioned in Table 1. Therefore, teachers must appear as a main stakeholder in the itineraries addressing these learning analytics approaches.

Table 1: List of recommendations for small-scale teacher-led learning analytics.

\begin{tabular}{|c|c|}
\hline Category & Recommendation \\
\hline Consent & $\begin{array}{l}\text { - If information is already being tracked, inform the students (or families) about it, } \\
\text { otherwise, ask for formal consent/agreement before data can be collected and/or } \\
\text { analyzed. } \\
\text { - Be explicit about what you might do with that information and, if possible, agree on } \\
\text { these possibilities with the students. } \\
\text { - Provide students with the option to update their digital dossiers and provide extra } \\
\text { (possibly qualitative) data but triangulate it to verify they do not "game" the system. } \\
\text { Data should be deleted when individuals no longer want it to be processed or when it } \\
\text { is no longer of use for its original purpose, as is the case with any other student data. } \\
\text { Reflect on the consequences that opting out of the analysis will have on the } \\
\text { participants (e.g., lack of feedback due to the lack of analytics) and inform them. }\end{array}$ \\
\hline Transparency & $\begin{array}{l}\text { - Be explicit about which data is collected, how it is interpreted, why, and how it will } \\
\text { affect the learning process. }\end{array}$ \\
\hline Access & $\begin{array}{l}\text { - Reflect on whether the students should access the data held about them, the analysis } \\
\text { of the data, the labels attached to them, and if so, in which format this information } \\
\text { should be provided to them. } \\
\text { - Consider the possibility of letting students correct the data stored about them. }\end{array}$ \\
\hline Responsibility & $\begin{array}{l}\text { - You are in charge of interpreting and validating the analyses as well as deciding what } \\
\text { to do based on the analytics (e.g., how to regulate, intervene, et cetera). }\end{array}$ \\
\hline Privacy & $\begin{array}{l}\text { - If some data must be anonymous, be sure it cannot be re-identified by contextual } \\
\text { information available to the users. } \\
\text { - If you use data from external sources, (e.g., Web } 2.0 \text { tools) be sure you can identify the } \\
\text { owner correctly, and that other ethical and privacy issues are not put at risk when } \\
\text { using those sources. }\end{array}$ \\
\hline Validity & $\begin{array}{l}\text { - The evidence obtained may be incomplete. Try to involve students (and families) in } \\
\text { order to increase its accuracy. }\end{array}$ \\
\hline Stewardship & $\begin{array}{l}\text { - Use the data needed for the analysis, nothing more. } \\
\text { Be sure that you comply with the data protection laws applicable in your region or } \\
\text { country. } \\
\text { - The data should be preserved, secured, and shared in the same way as any other } \\
\text { student data. }\end{array}$ \\
\hline $\begin{array}{l}\text { Avoiding } \\
\text { negative } \\
\text { impact }\end{array}$ & $\begin{array}{l}\text { - Consider that your analysis may produce unexpected results that prompt you to } \\
\text { intervene with the student. Which kinds of obligations will influence your actions? }\end{array}$ \\
\hline
\end{tabular}


The recommendations presented in Table 1 should be considered as a first attempt to structure reflection on ethical issues and logistical concerns in small-scale teacher-led learning analytics. They should be subject to refinement by their application to other cases, and by public discussion with experts in the field. These discussions might take place in the context of recent initiatives promoted by various governments (Hylen, 2015) that highlight the creation of policies and sharing practices of learning analytics in schools.

\section{$7 \quad$ CONCLUSION}

Interest in addressing ethical issues in learning analytics is starting to flourish in the form of ethical frameworks that guide codes of conduct for practitioners. These frameworks are useful instruments to structure the discussion and promote a more mature application of LA. However, current frameworks are focused on institutionally oriented higher education learning analytics. There is a need to extend and adapt these frameworks to the characteristics of different learning analytics approaches and educational contexts. Through two studies in primary and higher education contexts, this work has analyzed the ethical and privacy issues according to three different dimensions that may affect them: the overall learning analytics approach, the particular solution to learning analytics adopted, and the educational contexts where the analytics are applied.

The reflection presented in this work shows that the issues associated with smaller-scale teacher-led learning analytics require explicit consideration of the role of teachers as main actors in the application of the learning analytics processes. Aspects such as action and impact have a particular dimension, and are closer to general ethical issues related to classroom orchestration, where teachers play a crucial role. In this kind of educational context, it is necessary to analyze from an ethical point of view what kinds of actions should trigger the learning analytics output, how to proceed, and what the impact will be in terms of classroom management, intervention, regulation, and assessment.

We have shown how our approach to learning analytics, involving teachers from the very beginning in the design and enactment of the monitoring process helps to overcome issues that can appear in these smaller contexts, such as control of the analytics, awareness, et cetera. Teacher participation in the design of the monitoring process contributes to the development of "ethics by design" (parallel to the idea of "privacy by design" [Schaar, 2010] in the application of learning analytics solutions). According to this schema, the teacher is the one who defines the educational purposes of the analysis, reflects on the available data sources, improves the validity of the results (by adding new evidence from teachers and students), and is aware of the limitations of the results obtained.

Finally, the application of the approach to two educational contexts shows that reflection on learning analytics must take into account the specific ways of working associated with different contexts, including legal aspects that apply to the particular case of schools, where work with minors poses specific challenges and prompts the inclusion of families as new actors in the framework. The 
cloud-based tools that are becoming widespread at these educational levels do not deal with the information needs of LA and may pose legal and ethical problems related to data ownership and virtual identity, which are difficult to solve when working with minors. Not only LA, but also the wider technology-enhanced learning community faces a big challenge when providing appropriate tools to these educational levels.

This work is a first step towards the refinement and adaptation of ethical frameworks to the different approaches that currently coexist in the learning analytics field; for example, defining different itineraries depending on the approach to LA, so that practitioners can focus on the most important questions for their approach.

\section{ACKNOWLEDGMENTS}

This research has been partially funded by the European Commission's Lifelong Learning Programme, Project Number 526965-LLP-1-2012-1-GR-COMENIUS-CMP and by the Spanish Project, TIN2014-53199-C3-2-R. The authors would also like to thank the rest of the GSIC/EMIC Group at the University of Valladolid for their support and ideas, as well as teacher Daniel Mantilla Fernández and the students and families who participated in the study.

\section{REFERENCES}

Avouris, N., Fiotakis, G., Kahrimanis, G., Margaritis, M., \& Komis, V. (2007). Beyond logging of fingertip actions: Analysis of collaborative learning using multiple sources of data. Journal of Interactive Learning Research, 18(2), 231-250.

Blackwell, C. K., Lauricella, A. R., Wartella, E., Robb, M., \& Schomburg, R. (2013). Adoption and use of technology in early education: The interplay of extrinsic barriers and teacher attitudes. Computers \& Education, 69, 310-319. http://dx.doi.org/10.1016/j.compedu.2013.07.024

Dillenbourg, P. (2013). Design for classroom orchestration. Computers \& Education, 69, 485-492. http://dx.doi.org/10.1016/j.compedu.2013.04.013

Ebner, M., \& Schön, M. (2013). Why learning analytics in primary education matters! Bulletin of the Technical Committee on Learning Technology, 15(2), 14-17.

Ferguson, R. (2012). Learning analytics: Drivers, developments and challenges. International Journal of Technology Enhanced Learning, 4(5-6), 304-317. http://dx.doi.org/10.1504/IJTEL.2012.051816

Greller, W., \& Drachsler, H. (2012). Translating learning into numbers: A generic framework for learning analytics. Journal of Educational Technology \& Society, 15(3), 42-57. Retrieved from http://ifets.info/journals/15_3/4.pdf

Griffiths, D. (2012). The implications of analytics for teaching practice in higher education. CETIS Analytics Series, 1(10). Retrieved from http://publications.cetis.org.uk/wpcontent/uploads/2013/02/Analytics-for-Teaching-Practice-Vol-1-No-10.pdf

Hylen, J. (2015, October 1). Learning analytics in the Norwegian school system. LACE project archives [Web log post]. http://www.laceproject.eu/blog/learning-analytics-in-the-norwegian-schoolsystem/

Kay, D., Korn, N., \& Oppenheim, C. (2012). Legal, risk and ethical aspects of analytics in Higher ISSN 1929-7750 (online). The Journal of Learning Analytics works under a Creative Commons License, Attribution - NonCommercial-NoDerivs 3.0 Unported (CC BY-NC-ND 3.0) 
(2016). Learning analytics in small-scale teacher-led innovations: Ethical and data privacy issues. Journal of Learning Analytics, 3(1), 43-65. http://dx.doi.org/10.18608/jla.2016.31.4

education. CETIS Analytics Series, 1(6), 1-30.

Lim, C. P., Zhao, Y., Tondeur, J., Chai, C. S., \& Tsai, C. C. (2013). Bridging the gap: Technology trends and use of technology in schools. Journal of Educational Technology \& Society, 16(2), 59-68.

Pardo, A., \& Siemens, G. (2014). Ethical and privacy principles for learning analytics. British Journal of Educational Technology, 45(3), 438-450. http://dx.doi.org/10.1111/bjet.12152

Rodríguez-Triana, M. J., Martínez-Monés, A., \& Asensio-Pérez, J. (2011). Monitoring collaboration in flexible and personal learning environments. Interaction, Design and Architecture(s) Journal, Special Issue: Evaluating Educative Experiences of Flexible and Personal Learning Environments, 11(12), 51-63.

Rodríguez-Triana, M. J., Martínez-Monés, A., Asensio-Pérez, J. I., \& Dimitriadis, Y. (2012). Towards a monitoring-aware design process for CSCL scripts. In V. Herskovic, H. U. Hoppe, M. Jansen, \& J. Ziegler (Eds.), Collaboration and technology (Vol. 7493, pp. 223-236). Berlin: Springer. http://dx.doi.org/10.1007/978-3-642-33284-5_21

Rodríguez-Triana, M. J., Martínez-Monés, A., Asensio-Pérez, J. I., \& Dimitriadis, Y. (2013). Towards a script-aware monitoring process of computer-supported collaborative learning scenarios. International Journal of Technology Enhanced Learning, 5(2), 151-167. http://dx.doi.org/10.1504/IJTEL.2013.059082

Rodríguez-Triana, M. J., Martínez-Monés, A., Asensio-Pérez, J. I., \& Dimitriadis, Y. (2015). Scripting and monitoring meet each other: Aligning learning analytics and learning design to support teachers in orchestrating CSCL situations. British Journal of Educational Technology, 46(2), 330-343. http://dx.doi.org/10.1111/bjet.12198

Schaar, P. (2010). Privacy by design. Identity in the Information Society, 3(2), 267-274. http://dx.doi.org/10.1007/s12394-010-0055-x

Sclater, N. (2014). Code of practice for learning analytics: A literature review of the ethical and legal issues. Jisc. Retrieved from http://repository.jisc.ac.uk/5661/1/Learning_Analytics_ALLiterature_Review.pdf

Sclater, N. (2015, March 3). A taxonomy of the ethical and legal issues of learning analytics v0.1. Effective Learning Analytics. Jisc. [Web log post]. Retrieved from http://analytics.jiscinvolve.org/wp/2015/03/03/a-taxonomy-of-ethical-legal-and-logisticalissues-of-learning-analytics-v1-0/

Sclater, N., \& Bailey, P. (2015). Code of practice for learning analytics. Jisc. Retrieved from http://www.jisc.ac.uk/guides/code-of-practice-for-learning-analytics

Slade, S., \& Prinsloo, P. (2013). Learning analytics ethical issues and dilemmas. American Behavioral Scientist, 57(10), 1510-1529. http://dx.doi.org/10.1177/0002764213479366

Wise, A. F. (2014). Designing pedagogical interventions to support student use of learning analytics. Proceedings of the $4^{\text {th }}$ International Conference on Learning Analytics and Knowledge, 203211. http://dx.doi.org/10.1145/2567574.2567588 